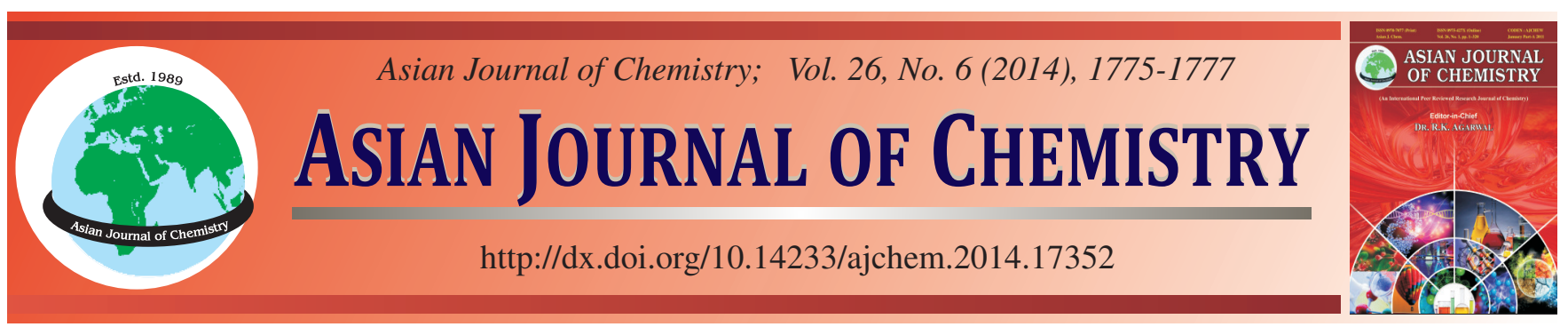

\title{
Foaming Effects, Interfacial Morphologies and Impact Toughness of Polypropylene Foam Composites Toughened by EOC- $g$-MAH $\dagger$
}

\begin{abstract}
JINIAN Y ANG $^{*}$ and CHUANG WANG
College of Materials Science and Engineering, Anhui University of Science and Technology, Huainan 232001, P.R. China

*Corresponding author: E-mail: yangjinian@163.com

Polypropylene/short glass fiber (PP/SGF) and PP/SGF/ethylene-1-octene (EOC) foam composites, with maleic anhydride grafted EOC (EOC- $g-\mathrm{MAH})$ as compatilizer and impact modifier, were prepared. The cellular structures, interfacial morphologies and impact toughness were investigated. Results show that EOC- $g$-MAH is preferred to improve the foaming effects of the sample. Also, it is confirmed to be an effective plasticizer in the PP/SGF foam composites. Moreover, the highest impact toughness is obtained by the case of EOC-g-MAH modified PP/SGF/EOC. SEM observation reveals that interfacial interaction between short glass fiber and matrix is enhanced and EOC$g$-MAH also facilitates the compatibility of ethylene-1-octene and polypropylene.
\end{abstract}

Keywords: Polypropylene, Foam composites, Ethylene-1-octene, Toughen.

\section{INTRODUCTION}

Nowadays, polypropylene foam has been widely used in many industrial fields because of its excellent chemical and mechanical properties. However, the facts of the intrinsic shortcomings of polymeric foam and poor impact resistance of polypropylene itself make it difficult for the major development in structural materials requesting high strength, stiffness and toughness. Many attempts have been carried out to design and develop the high performance polypropylene foams and the introduction of short fiber and elastomer of making polypropylene foam composites were confirmed as the most effective methods to strengthen and toughen the polypropylene foams, respectively ${ }^{1-3}$. Moreover, researchers also reported that the addition of $5 \%$ interfacial compatilizer could lead to an improvement up to $80 \%$ in the mechanical properties ${ }^{2}$. Therefore, in this work, the maleic anhydride grafted ethylene-1-octene (EOC- $g$-MAH) was utilized as impact modifier and compatilizer into polypropylene/short glass fiber (PP/SGF) and polypropylene/short glass fiber/ethylene-1-octene (PP/SGF/EOC) foam composites and then the evolution of cellular structures, interfacial morphologies and impact toughness were investigated.

\section{EXPERIMENTAL}

Polypropylene [PPH-XD-140, melt flow rate (MFR) = $14 \mathrm{~g} / 10 \mathrm{~min}$ ] was bought from Jinling Petrochemical Co., Ltd,
China. EOC (Engage 8180, MFR =0.5 g/10 min) was purchased from Dow Chemical Company (USA). EOC- $g$-MAH (HD800E) was the product of Nanjing Huadu Co., Ltd., China. SGF was provided by Nanjing Fiberglass R \& D Institude, China, pretreated with silane coupling agent (A1100). Compounded foaming agent consisted of azodicarbonamide, $\mathrm{ZnO}$ and $\mathrm{SiO}_{2}$ was pre-mixed homogeneously with a mass ratio of 1: 0.1: 0.15 .

All raw materials used were dried separately in ovens for $24 \mathrm{~h}$ at $60{ }^{\circ} \mathrm{C}$. Then the premixed mixtures were fed into a CM-30 single-screw extruder with the temperature profiles of $160-180^{\circ} \mathrm{C}$ for melt compounding and chopped into granules. The dried granules were mould compression foamed at 170$175^{\circ} \mathrm{C}$ for $2.0-2.5 \mathrm{~h}$ to form polypropylene foam composites with densities of $0.45 \pm 0.05 \mathrm{~g} / \mathrm{cm}^{3}$. The PP/SGF and PP/SGF/ EOC foam composites with and without EOC- $g$-MAH were prepared and they were set as PP/SGF (100/20), PP/SGF/EOC$g$-MAH (100/20/5), PP/SGF/EOC (100/20/20) and PP/SGF/ EOC/EOC- $g$-MAH (100/20/20/5), respectively.

The un-notched impact test was performed in a XJ-300A impact tester at room temperature with a sample dimension of $75 \mathrm{~mm} \times 15 \mathrm{~mm} \times 15 \mathrm{~mm}$. Surface energies were determined using a CCA-100 contact angle tensiometer at room temperature with distilled water as reference liquids. The surface energy $(\gamma)$ was composed of the dispersion $\left(\gamma^{d}\right)$ and polar $\left(\gamma^{p}\right)$ components and the interfacial energy $\left(\gamma_{\mathrm{AB}}\right)$ was calculated

$†$ Presented at The 7th International Conference on Multi-functional Materials and Applications, held on 22-24 November 2013, Anhui University of Science \& Technology, Huainan, Anhui Province, P.R. China 
according to the reference ${ }^{4}$. The impact fractured surfaces were selected and coated with a thin layer of gold and then examined with SEM (JSM-5610LV). Approximate 50-100 cells from the micrographs were then analyzed using Image-Pro Plus Software to obtain the average cell diameter, bubble-size distribution and variance of cell size, which, together with the cell density calculated by reported method ${ }^{5}$, were used to determine the foaming effect of polypropylene foam composites.

\section{RESULTS AND DISCUSSION}

Cellular structures: Fig. 1 shows that the cellular morphologies of samples seem barely changed with EOC- $g$-MAH, still exhibiting closed spherical-like bubbles. The PP/SGF/EOC shows a more competitive cellular structure than PP/SGF, while the best foaming effect is obtained in Fig. 1(c) with the evidences of minimum values of average cell diameter $(0.438 \mathrm{~mm})$ and variance (0.0194) and maximum cell density $(11,489$ cells/ $\mathrm{cm}^{3}$ ) (Table-1). The possible reason would be that the EOC- $g$ MAH plays a great role in strengthening the interfacial bonding between SGF and matrix and thus the dispersion of SGF in matrix should be improved. As a result, the melt strength should be improved due to the enhanced interactions amongst the SGF and matrix, facilitating the bubble stabilization. However, the EOC- $g$-MAH seems to have no positive effect on the foaming effect in ternary system (Fig. 1d), although which is still better than PP/SGF (Fig. 1a).
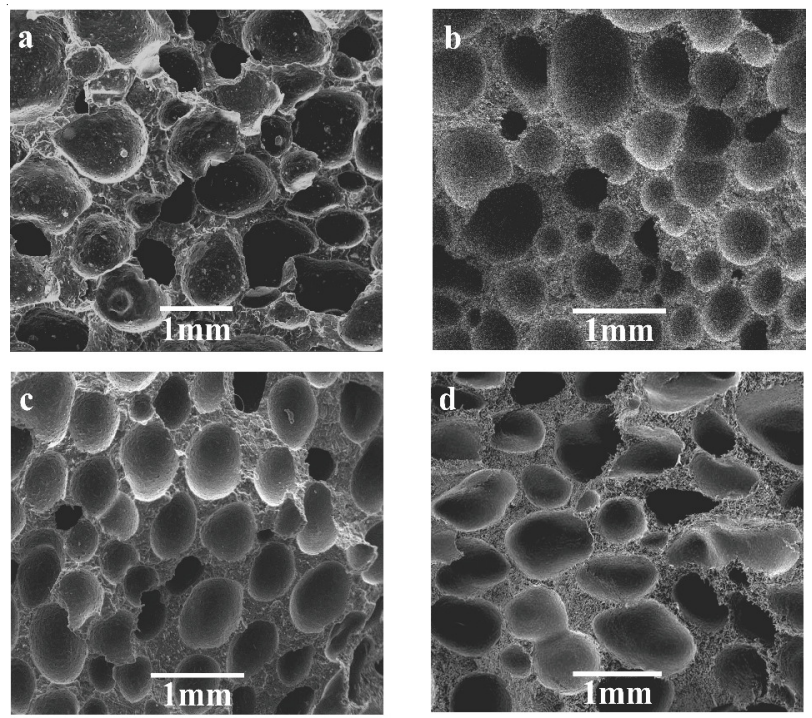

Fig. 1. SEM micrographs of polypropylene foam composites: (a) PP/SGF; (b) PP/SGF/EOC; (c) PP/SGF/EOC- $g$-MAH; (d) PP/SGF/EOC/ EOC- $g$-MAH

Impact toughness: Fig. 2 showed that the impact toughness of PP/SGF is lowest. With adding $5 \%$ EOC- $g$-MAH, the impact toughness of PP/SGF/EOC- $g$-MAH is heightened up to $58 \%$ improvement compared with $\mathrm{PP} / \mathrm{SGF}$, whereas the same EOC- $g$-MAH content just leads to less than $5 \%$ improvement for PP/SGF/EOC/EOC- $g$-MAH. This indicates a glaring difference in the toughening efficiency of EOC- $g$-MAH on the binary and ternary systems. It should be mainly ascribed to the great improvements in the foaming effect of PP/SGF/ EOC- $g$-MAH and then much more energy could be consumed before materials crashed to pieces. Moreover, the remarkable

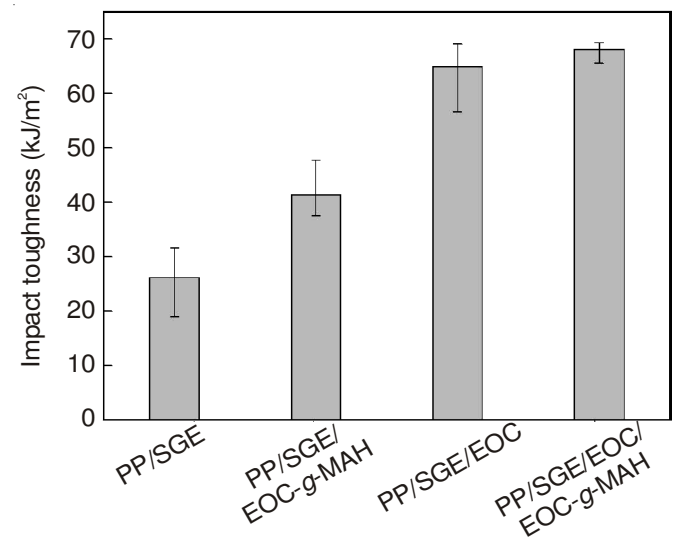

Fig. 2. Impact toughness of polypropylene foam composites

\begin{tabular}{lccc}
\multicolumn{5}{c}{ TABLE-1 } \\
MORPHOLOGICAL PARAMETERS OF PP FOAM COMPOSITES \\
\hline \multicolumn{1}{c}{ Samples } & $\begin{array}{c}\text { Diameter } \\
(\mathrm{mm})\end{array}$ & $\begin{array}{c}\text { Cell density } \\
\left(\mathrm{cells} / \mathrm{cm}^{3}\right)\end{array}$ & Variance \\
\hline PP/SGF & 0.646 & 3,581 & 0.0783 \\
PP/SGF/EOC & 0.468 & 8,692 & 0.0373 \\
PP/SGF/EOC- $g$-MAH & 0.438 & 11,489 & 0.0194 \\
PP/SGF/EOC/EOC- $g$-MAH & 0.558 & 5,139 & 0.0417 \\
\hline
\end{tabular}

elastomer-toughening effect derived from elastomer phase should be another key point for the tremendous increase in impact toughness.

Interfacial morphologies: Fig. 3 showed the SGF was randomly distributed and the elastomeric phases acted as dispersed particles in the impact fracture surfaces of polypropylene foam composites. It is clearly seen from Fig. 3(a) and 3(b) that there were few residual material parts left on the SGF surface, which indicates that failure occurred mainly at the fiber-matrix interface due to the poor interfacial adhesion. In contrast, the interfacial bonding between SGF and matrix is improved significantly with EOC- $g$-MAH added shown in Fig. 3(c) and 3(d). This enhanced interfacial structure between SGF and matrix would be another possible contribution for the increase toughness, because it should consume more energy during the fiber pulled out from the matrix. Moreover, from Fig. 3(c), it is interestingly found that the EOC-g-MAH particles not only dispersed around the SGF, but also adhered firmly onto the surfaces of drawn fibers. However, a different morphology was exhibited in Fig. 3(d), i.e., the elastomeric particles are not existed as the same typical droplet-matrix structure as Fig. 3(b) shown with clear boundary, but mainly embedded into polypropylene matrix with blurry interfaces and almost no isolated bare particles appeared on the fracture surface. Nevertheless, this embedded structure of elastomeric particles in matrix might result in the sacrifice of interaction between SGF and EOC and lead to the negative effect on the cellular structures in Fig. 3(d).

To understand this difference of morphologies between Fig. 3(c) and 3(d), the thermodynamic parameters should be proposed and compared. Table- 2 listed the surface energies $(\gamma)$ of the materials under investigated and the calculated interfacial energy $\left(\gamma_{\mathrm{AB}}\right)$ of all of the possible pairs. From Table2 , the highest $\gamma_{\mathrm{AB}}$ for SGF/EOC means the interfaces hard to form and yields segregated status between SGF and EOC. However, the pair of SGF/EOC- $g$-MAH has the stronger tread 


\begin{tabular}{|c|c|c|c|c|c|}
\hline \multirow{3}{*}{ Materials } & \multicolumn{4}{|c|}{$\begin{array}{l}\text { TABLE-2 } \\
\text { E ENERGY }(\gamma) \text { AND CALCULATED } \gamma_{A B}\end{array}$} & \multirow{3}{*}{$\gamma_{\mathrm{AB}}\left(\mathrm{mJ} / \mathrm{m}^{2}\right)$} \\
\hline & \multicolumn{3}{|c|}{ Surface energy $\left(\mathrm{mJ} / \mathrm{m}^{2}\right)$} & \multirow{2}{*}{ Possible pairs } & \\
\hline & $\gamma$ & $\gamma^{d}$ & $\gamma^{p}$ & & \\
\hline $\mathrm{PP}$ & 28.04 & 27.24 & 0.80 & $\mathrm{SGF} / \mathrm{PP}$ & 6.67 \\
\hline SGF & 32.07 & 23.54 & 8.53 & PP/EOC & 0.83 \\
\hline EOC & 21.71 & 21.23 & 0.48 & SGF/EOC & 7.31 \\
\hline EOC- $g$-MAH & 17.51 & 15.61 & 1.90 & SGF/EOC- $g$-MAH & 5.82 \\
\hline- & - & - & - & PP/EOC- $g$-MAH & 3.60 \\
\hline - & - & - & - & EOC/EOC- $g$-MAH & 1.70 \\
\hline
\end{tabular}
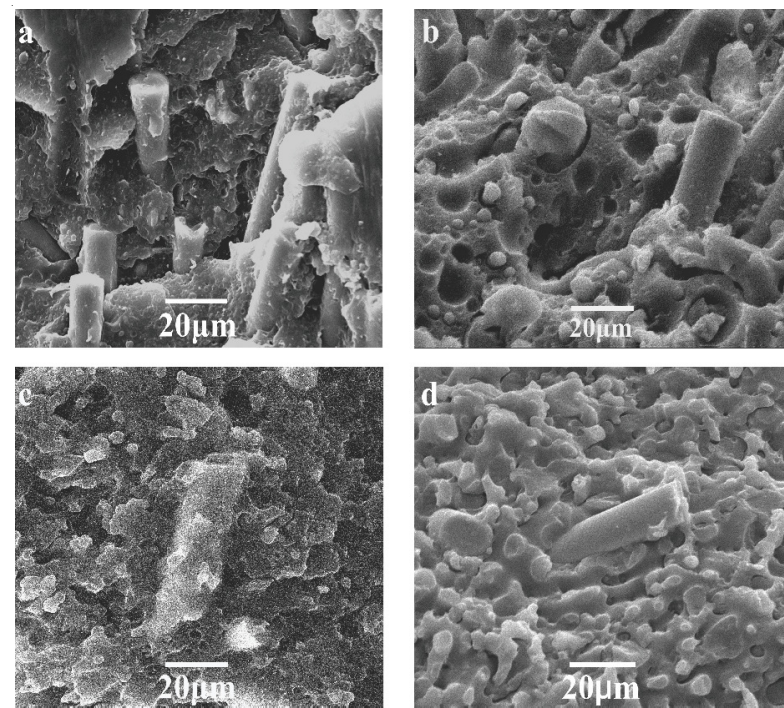

Fig. 3. SEM micrographs showing impact fracture surfaces of (a) PP/SGF; (b) PP/SGF/EOC; (c) PP/SGF/EOC- $g$-MAH; (d) PP/SGF/EOC/ EOC- $g$-MAH

of phase formation proved by the lower $\gamma_{\mathrm{AB}}$, as could be used to explain the enhanced interfacial interaction between SGF and EOC-g-MAH in Fig. 3(c). Moreover, a very important part played by the amidation reaction of anhydride groups with amino groups ${ }^{6}$, which is taken place between SGF and EOC$g$-MAH, would never be ignored and it also could be used for explaining the formation of particle-adhered structures in Fig. 3(c). However, the strongest trend to form phase morphologies of PP/EOC and EOC/EOC- $g$-MAH (with only 0.83 and 1.70 $\mathrm{mJ} / \mathrm{m}^{2}$, respectively) might stop the possible chemical reaction between EOC- $g$-MAH and SGF from taking effect and then the embedded structures of elastomeric droplets into matrix are formed shown in Fig. 3d.

\section{Conclusion}

The introduction of EOC- $g$-MAH could improve the foaming effect of PP/SGF foam composites significantly and lead to an improvement up to $58 \%$ on the impact toughness; whereas much less effect is shown in the case of EOC- $g$-MAH modified PP/SGF/EOC. SEM observation demonstrates that there are EOC- $g$-MAH particle-adhered structures in the PP/ $\mathrm{SGF} / \mathrm{EOC}-\mathrm{g}$-MAH. However, the EOC- $g$-MAH is preferred to facilitating the compatibility of EOC and polypropylene, exhibiting a particle-embedded configuration in the PP/SGF/ EOC modified with EOC- $g$-MAH.

\section{REFERENCES}

1. S.W. Zhang, D. Rodrigue and B. Riedl, Polym. Compos., 26, 731 (2005).

2. A.K. Bledzki and O. Faruk, Cell. Polym., 21, 417 (2002).

3. J.N. Yang, Z.Q. Li and J.S. Liu, E-Polymers, 076, 1 (2011).

4. S. Wu, Polymer Interface and Adhesion, CRC Press, New York (1982).

5. M. Okamoto, P.H. Nam, P. Maiti, T. Kotaka, T. Nakayama, M. Takada, M. Ohshima, A. Usuki, N. Hasegawa and H. Okamoto, Nano Lett., 1, 503 (2001).

6. A. Zheng, H.G. Wang, X.S. Zhu and S. Masuda, Compos. Interfaces, 9, 319 (2002). 